\title{
Musical hallucinations associated with acquired deafness
}

\author{
THOMAS A HAMMEKE, MICHAEL P MCQUILLEN, BERNARD A COHEN \\ From the Department of Neurology, Medical College of Wisconsin, Milwaukee, Wisconsin, USA
}

SUMMARY Two patients with auditory hallucinations beginning after a long history of progressive bilateral hearing loss were studied. The hallucinations included both unformed (tinnitus and irregular sounds of varying pitch and timbre) and formed (instrumental music, singing and voices) components, and were repetitive. They were affected by ambient noise levels; their content and speed were influenced by attentional and intentional factors. There was no evidence of global dementia, nor of epileptogenic or psychiatric disturbance. A combination of peripheral and associated central "disinhibition" may be responsible for the occurrence of such hallucinations.

In recent years several instances of formed auditory hallucinations associated with acquired deafness have been reported. ${ }^{1-3}$ We describe two further cases.

\section{Case reports}

Case One: A 75-year-old, right-handed, retired female school teacher complained of annoying musical hallucinations. The first occurred four months earlier, shortly after discontinuing an antibiotic medication for a sinus infection. She was awakened during the night by the "music," which lasted one hour. The music was loud and vivid, prompting her to search her living quarters for the source. A similar experience occurred the following night and throughout the subsequent day. During the following three weeks, the episodes increased in frequency until they were a constant experience except during periods of sleep, active conversation or mental operation. She described the experience to include both familiar and unfamiliar ("nonsense") melodies. Familiar melodies were ballads and religious hymns learned in childhood. Often the melodies were played by a single instrument (for example, guitar or chimes) and sung by a baritone voice; occasionally they were sung by a choir with orchestral accompaniment. The melodies were repetitive (line or verse) and present in both ears. Intensity increased when she was mentally inactive

Address for reprint requests: Thomas A Hammeke, PhD, Section of Neuropsychology, Medical College of Wisconsin, 9001 Watertown Plank Road, Milwaukee, Wisconsin 53226, USA.

Received 9 August 1982 and in revised form 25 January 1983 Accepted 7 February 1983 and ambient noise levels were low. She reported an ability to replace an ongoing musical passage with another, or alter its speed, via subvocalisation of the desired song or speed. In addition, she reported a gradual, progressive hearing loss over several years, that prompted her to purchase a hearing aid two years earlier. While hearing was impaired, it was adequate for conversation (with the aid of the hearing aid and occasional lip reading).

Audiometric testing revealed moderate, bilateral sensorineural hearing loss (51 db AD, $56 \mathrm{db}$ AS); computed tomographic (CT) scan showed mild diffuse atrophy with no focal areas of enhancement; an electroencephalogram (EEG) found mixed dysrhythmic complexes throughout central and posterior regions bilaterally; brainstem auditory evoked potential (BAEP) studies demonstrated increased interpeak latencies with stimulation to the left ear; somatosensory evoked potentials (SEP) showed diminished amplitude over the left cerebrum; and visual pattern-shift evoked potentials (VEP) were normal. Intellectual testing found superior intellectual (Full Scale IQ = 121 , Verbal IQ $=131$, Performance $\mathrm{IQ}=106$ ) and mnemonic skills (Wechsler Memory Scale Memory Quotient $=124$ ). Individual subtests contributing to IQ indices were in average ranges uniformly (age-corrected scale scores were greater than 11), except for two measures of visuo-constructional skills (Block Design and Object Assembly: age corrected scale scores of 10 and 7 respectively), and for a measure of logical sequential reasoning (Picture Arrangement: scale score of 8). Extensive neuropsychological testing failed to find any evidence of significant deficits in temporal orientation, immediate or delayed memory for verbal or nonverbal materials, praxis, graphomotor constructional and calculation skills, lan- 
guage functions, or motor skills. One exception to this was moderate impairment in speech sound discrimination. Although difficulties in speech sound discrimination were thought consistent with the hearing loss, they occurred in the context of normal performance in discrimination of rhythm, pitch and melody. Also, moderate impairment in graphaesthesia (tactile symbol recognition) was found bilaterally, but more pronounced on the right hand. When the same test was administered two years later, the lefthand performance had deteriorated to a degree where no asymmetry was present. On both testing occasions, no difficulty in tactile finger identification was observed. Personality testing with the Minnesota Multiphasic Personality Inventory yielded a profile entirely within normal limits.

Case Two. An 80-year-old, right-handed nun and retired school teacher reported a three year history of auditory hallucinations. She had been deaf in the right ear since a mastoidectomy some $\mathbf{4 0}$ years earlier. She had been troubled by recurrent ringing and buzzing in her left ear when, in the summer of 1972, she was exposed to an "extremely intense noise (traffic)." Several hours later she became aware of an intense noise in her head "like a boiler factory." This was followed by the perception of someone singing "Jingle Bells." Since that time she described a constant experience of a variety of repetitive sounds. At times the sounds were simply formed ("rumbling noises"). At other times words or phrases, that she had most recently read or been told, were repeated. In the first year after onset, melodies were common. In subsequent years voices repeatedly calling a name she went by as a child, or repetition of a brief religious prayer (for example, "Lord have mercy") became more frequent. The intensity of hallucinations was greatest during periods of silence and when her hearing aid was turned down. A few brief episodes of loss of consciousness without apparent convulsive behaviour had occurred several years earlier; these were thought to be secondary to glucose intolerance. Audiometric testing showed profound right ear $(100 \mathrm{db})$ and moderate left ear $(50 \mathrm{db})$ sensorineural hearing loss (but with a hearing aid, the patient was able to converse easily); CT scan showed mild cerebral atrophy; and repeated EEGs taken over the course of several years showed sharp theta waves over the temporal regions bilaterally with questionable left temporal spiking. Audiovisual monitoring with the EEG found no correlation between the sharp waves and the patient's symptom report. BAEP, SEP and VEP studies were normal. Limited neuropsychological testing was done. She was fully alert and oriented and achieved average scores on the Peabody Picture Vocabulary Test (PPVT IQ $=116$ ) and the Wechsler Memory Scale $(M Q=100)$. Despite these average scores, performance on a more specific measure of nonverbal memory (Benton Visual Retention Test) was severely impaired. Also, she was unable to learn a sequence of eight digits in twelve learning trials. Language screening was unremarkable, except for moderate difficulties in speech sound discrimination, accompanied by comparable difficulties in pitch, rhythm and melody discrimination. Verbal spontaneity (Controlled Word Association Test) was within the average range. No evidence of ideomotor apraxia was found. Figure drawings showed mild configural distortions and occasional perseverative tendencies. Serial additions were performed poorly. Fine motor speed and dexterity measures were within normal limits and symmetric. Occasional right-sided tactile sensory extinctions occurred with double simultaneous stimulation. Occasional errors in tactile finger identification were found bilaterally. In testing graphaesthesia, the patient had moderate difficulty in recognising numbers traced on the finger tips of either hand with the errors occurring more frequently on the right hand by a ratio of 3:1. No formal personality testing was done; however, no evidence of psychiatric disturbance was observed nor discerned from medical records or history.

\section{Discussion}

The features of these patients are consistent with those of other reported cases. ${ }^{1-4}$ In both patients the non-threatening hallucinations began abruptly after several years of progressive deafness, and at a time when a decrement in hearing may have occurred. The hallucinations included both formed and unformed components of a repetitive nature. Often, the formed hallucinations were detailed and vivid. Frequently, they represented musical passages or voices first heard in childhood. Generally, the experience was constant, but intensified during periods of inactivity and under conditions of reduced ambient noise levels. In neither patient was there any clear evidence of psychosis, drug withdrawal or seizures. Both patients were annoyed by the hallucinations, particularly by the repetitive features; but they appeared to develop a measure of tolerance for them. Interestingly, and similar to other reports, ${ }^{1-2}$ both patients indicated that they could replace ongoing hallucinations wilfully with songs or prose of their own choosing via concentration or subvocalisation. In both patients the hallucinations persisted over several years in the face of treatment with anti-convulsant, anti-psychotic and vitamin supplement therapy, although each of these may have had a minor attenuating effect. The condition may well be permanent, as indicated by reports of cases having the hallucinations for more than two decades. ${ }^{3}$

Neuropsychological studies failed to find evidence of global dementia, although the second patient manifested some evidence suggestive of mild, probably diffuse cerebral dysfunction (impairment in memory and learning tasks that are out of keeping with expected levels for her age ${ }^{5}$ ). Of perhaps greater interest are findings suggestive of more regional brain pathology. Both cases performed poorly on right-sided tactile-perceptual measures, a finding consistent with abnormal SEPs in the first patient implicating possible left parietal dysfunction. Also, the question of more localised right hemisphere dysfunction is raised in each case. The first patient 
showed mild reductions in visual-constructional and logical sequential reasoning. Non-verbal memory was more severely impaired than verbal memory in the second patient.

Investigators disagree about the anatomical substrate for the phenomenon of hallucinations. Some ${ }^{4-8}$ maintain that neural or end organ disease alone is sufficient to produce musical hallucinations or other similar phenomena (for example visual hallucinations associated with blindness). These investigators ${ }^{18-10}$ postulate "sensory deprivation" as the primary neurophysiological mechanism. Under conditions of reduced sensory input, perception-bearing circuits are disinhibited and perceptual traces "released," producing a re-experience of a single or combination of perceptions. Other investigators argue that a combination of peripheral and central dysfunction is required. ${ }^{11-12}$ The two-factor theorists draw support from the fact that hallucinations of this sort occur most frequently in elderly populations where the incidence of brain pathology is increased. Of fifteen cases described in several reports ${ }^{1-2414}$ including our own, the mean age at onset was 65 years (range $=49-89$ ). More direct evidence of central dysfunction is provided by electrophysiological and radiographic studies in a few cases. EEG abnormalities were present in three of five cases studied. ${ }^{1-2}$ CT evidence of atrophy was present in all three cases studied. ${ }^{1}$ Further, the neuropsychological and evoked potential studies in our cases were suggestive of mild localised cortical dysfunction, perhaps on a background of mild generalised atrophy. Whether the central dysfunction implied by these findings is critical, contributory, or incidental to the phenomenon is not known.

\section{References}

' Miller TC, Crosby TW. Musical hallucinations in a deaf elderly patient. Ann Neurol 1979;5:301-2.

${ }^{2}$ Ross ED, Jossman PD, Bell B, Sabin T, Geschwind N. Musical hallucinations in deafness. JAMA 1975;231:620-2.

${ }^{3}$ Ross ED. Musical hallucinations in deafness revisited. JAMA 1978;240:716.

${ }^{4}$ Rozanski J, Rosen H. Musical hallucinosis and otosclerosis. Confin Neurol 1952;12:49-54.

${ }^{5}$ Benton AL, Eslinger PJ, Damasio AR. Normative observations on neuropsychological test performances in old age. J Clin Neuropsychol 1981;3:33-42.

${ }^{\circ}$ Hecaen H, Albert ML. Human Neuropsychology. New York: Wiley, 1978.

${ }^{7}$ Weinberger L, Grant F. Visual hallucinations and their neuro-optical correlates. Arch Opthalmol 1940;23:166-9.

${ }^{8}$ West LJ. A clinical and theoretical overview of hallucinatory phenomena. In: Siegel RK, West LJ, eds., Hallucinations: Behavior, Experience and Theory. New York, Wiley, 1975, 297-311.

${ }^{9}$ Cogan D. Visual hallucinations as release phenomena. Albrecht Von Graefes Arch Klin Exp Opthalmol 1973;188:139-50.

${ }^{10}$ Brust JCM, Behrens MM. "Release hallucinations" as the major symptoms of posterior cerebral artery occlusion: a report of two cases. Ann Neurol 1977;2:432-6.

" McNamara ME, Heros RC, Boller F. Visual hallucinations in blindness: the Charles Bonnet syndrome. Int $J$ Neurosci, 1983.

${ }^{12}$ Burgermeister R, Tissot R, de Ajuriaguerra J. Les hallucinations visuelles des opthalmopathes. Neuropsychologia 1965;3:9-38.

${ }^{13}$ Rhein JHW. Hallucinations of hearing and diseases of the ear. NY Med J 1913;97:1236-8. 